\title{
Investigating Saudi Learners' Preferences for Giving and Receiving Macro and/or Micro Level Peer Feedback on Their Writing
}

\author{
Suliman Mohammed Alnasser ${ }^{1} \&$ Hesham Suleiman Alyousef ${ }^{1}$ \\ ${ }^{1}$ Department of English Language and Litrature Faculty of Arts, King Saud University, Riyadh, Saudi Arabia \\ Correspondence: Hesham Suleiman Alyousef, P.O. Box 50574, Riyadh 11533, Saudi Arabia. Tel: \\ 96-655-300-0412. E-mail: hesham@ksu.edu.sa
}

Received: February 15, 2015 Accepted: April 1, 2015 Online Published: May 28, 2015

doi:10.5539/elt.v8n5p57

URL: http://dx.doi.org/10.5539/elt.v8n5p57

\begin{abstract}
Several studies have addressed the subject of the preferences of L2 student-writers for receiving teacher feedback (FB) on macro level features (feedback related to meaning) and micro level features (feedback related to surface level issues); however, none of these have investigated their preferences when it comes to giving and receiving peer feedback (PF). In the present study, two forms of the peer feedback technique were introduced in two consecutive phases: in phase one the students practised providing both macro and micro peer feedback on five essays, while in phase two they provided only macro feedback to their fellow students while the teacher supplied the micro feedback. The participants were 41 EFL undergraduate Saudi students undertaking an English programme at a Saudi higher tertiary institution. The study adopted a one-group design for the data collection. A mixed method approach was employed using pre-, mid- and post-questionnaires, mid- and post-interviews, and also recording verbal protocol sessions while the participants provided peer feedback to one another. The findings of the study suggested that the participants had a preference for giving and receiving peer feedback on both levels. However, it also appeared that their responses may have been affected by the way they had been taught in the past.
\end{abstract}

Keywords: collaborative learning, L2 writing, macro and micro level features, peer feedback, Saudi learner preference

\section{Introduction}

Various studies have examined learners' preferences for receiving teacher feedback (FB) on their writing (e.g., Leki, 1991), with the majority of these studies focusing on teacher FB on micro level writing features (i.e., surface level features such as grammar and proper use of vocabulary). The investigation of this area is normally appreciated by researchers who argue for or against the provision of error correction (i.e., micro FB). However, teacher FB is only one among the variuos FB sources available in L2 writing. Peer feedback (PF) technique, for example, is a feedback source that is commonly used in higher education because of its positive impact within the language learning context. To the best of our knowledge, no previous studies have investigated learner preferences for receiving macro (i.e., meaning level features such as idea development and the organisation of a written text) and/or micro level PF. The investigation of this area has the potential to produce findings that may help to clarify some of the concerns that have been reported relating to the PF technique (for instance, it has been suggested that learners do not accept most of the PF they receive (Min, 2006; Zhao, 2010).

\section{Review of Relevant Literature}

There have been many arguments both for and against the provision of feedback on micro level writing features; however, to the best of our knowledge no consensus has been reached on this matter among researchers. However, those who disagree with the provision of micro feedback present some compelling arguments. For example, micro feedback can be discouraging and unhelpful (e.g., Truscott, 1999). Also, it can be time-consuming and ineffective and can shift the teacher's attention away from more important concerns in writing-i.e., macro level features (Truscott, 1999). Additionally, others have concluded in their studies that micro FB can be harmful to the learning process (e.g., Fazio, 2001; Kepner, 1991; Sheppard, 1992). Learners may tend, for example, to simplify and shorten their writing in an attempt to avoid making errors. Moreover, an empirical study by Sheppard (1992) found that responding to macro level issues is more likely to improve grammatical accuracy than responding only to micro level issues. Finally, it has been suggested that learners are 
less capable of self-editing lexical errors (Ferris, 2004).

This dispute both for and against the provision of feedback on the micro level writing has encouraged several researchers to investigate learners' preferences for receiving teacher FB on each level (i.e., macro and micro levels), some of whom have reported that both levels of FB are preferred, with an occasional preference for micro FB (e.g., Hedgcock \& Lefkowitz, 1994; Leki, 1991; Ferris, 2004). Those who argue for the provision of micro FB use this evidence to support their claims and imply that meeting learners' preferences can yield positive learning experience. This in turn raises the question of whether or not we should consider learners' preferences in relation to learning. In this regard, Truscott (1999) suggests that learners' preferences should not be taken into consideration if they are unhelpful to their learning, while Bitchener and Ferris (2012) encourage teachers to consider learners' preferences, as this may allow more learner-involvement. However, more research is needed in this area to prove whether or not there is a relationship between meeting learners' preferences on the one hand and learning outcomes on the other.

In an L2 context, learners may seem to have preference for micro FB, since the provider of this type of feedback is a reliable source (i.e., the teacher) and learners are likely to have a desire to overcome their weaknesses at the micro level in particular; however, their opinions may vary when it comes to receiving FB from their fellow students. As mentioned above, there is a view that micro FB may have a negative impact on learners even when an educational expert provides it; therefore, this negative impact may increase when micro FB is given by an unreliable source (e.g., the learner). As a result, the PF technique may not lead to better learning, and may lead to negative results. Therefore, since this area has been neglected in the literature, the present study aims to investigate learners' preferences for receiving macro and micro PF in order to provide deeper insights for the use of PF technique in L2 writing classrooms.

Having reviewed previous PF studies and identified the research gap in this reespect, next we present the research design and methodology of the present study.

\section{Methodology}

This study was part of a larger project that introduced the PF technique in two different forms (the conventional form and a new form) to learners in a higher educational context. A quasi-action research approach was employed in the study using a one-group design for data collection. The participants were 41 male EFL Saudi undergraduate learners undertaking an English language programme. The participants were in their final year and shared a similar educational background. All participants agreed to participate in the study and signed a consent form. The researcher/teacher took over an advanced writing module and collected the data during the whole academic semester- i.e., data were collected for a period of 15 weeks. There were three 1-hour sessions a week. The aim of the study was to answer the following research questions:

1) Do learners have a preference for receiving PF on micro and/or macro level features?

2) Do learners preference change after exposure to each form of the peer feedback technique?

3) What reasons are there for their preferences?

In order to answer the research questions, the researcher designed the study in a way that allowed the same participants to practise using the conventional PF technique (i.e., providing PF on macro and micro level features) for a period of time, and then to practise using a new form in which the participants were allowed to provide only macro PF and prohibited from providing micro PF; the provision of micro FB was deemed to be the teacher's task during that period. A mixed method approach was used in the research, combining both qualitative and quantitative methods. Three stages of identical questionnaires and two stages of identical semi-structured interviews were administered in order to investigate their views and to measure any changes in these views before and after practice with each treatment, i.e. the conventional PF technique (T1) and the new suggested form of the PF technique (T2). Additionally, verbal protocol (a think-aloud method) recording sessions were carried out.

A five-point Likert scale was employed, incorporating the commonly used categories for rating scales: (1) strongly disagree, (2) disagree, (3) not sure, (4) agree and (5) strongly agree. Questionnaire items were:

- I appreciate the way that my classmates give me written comments on my essays.

- When my classmate gives me feedback, I want to see comments indicating errors in spelling and vocabulary.

- When my classmate gives me feedback, I object to seeing comments indicating errors in grammar and punctuation.

- When my classmate gives me feedback, I want to see comments on the organisation of my essay. 
- When my classmate gives me feedback, I object to seeing comments on the ideas I have expressed.

- I prefer focusing only on macro issues when giving and receiving peer feedback.

- I prefer focusing on both macro and micro issues when giving and receiving peer feedback.

- Focusing on one level encourages participation in PF.

With regard to the qualitative aspect of the study, 11 participants volunteered to be interviewed and were asked the following question:

- Do you prefer giving and receiving FB based on macro and micro levels? Or just one level? Why?

With regard to the verbal protocol sessions, a few sessions were audio-recorded while the students were giving and receiving PF. We observed and interrupted the conversations, seeking clarification of any unusual phenomena occurring during the one-to-one PF sessions.

Each volunteer pair of students sat with the researcher in an isolated room, taking turns at giving and receiving the feedback (in that one student gave PF and the other discussed/negotiated this PF) using their first languge, Arabic, while the researcher observed and recorded each turn. They were informed that there would be interruptions by the researcher in order to seek clarifications about any observed phenomenon.

\subsection{Practical Procedures}

The design of the study consists of two consecutive phases, as described next.

\subsubsection{Phase One}

In this phase the participants were given a pre-questionnaire, and then they were trained on how to use the PF technique employing Min's (2006) module, in which the teacher demonstrates aloud how to provide FB. Next, the participants wrote an argumentative essay and generated first drafts. After that they were assigned to work with peers who possessed the same level of language proficiency (this was determined by the researcher by referring back to their academic records). They exchanged essays and started taking turns in proving PF to each other using the evaluation form, which was adapted from Alhazmi and Schofield (2007) and which provides detailed questioning on macro and micro writing features. After exchanging comments, the participants developed their second drafts. They wrote four additional argumentative essays, exchanging comments and developing two drafts on each topic. Therefore, the participants practised providing PF on five argumentative essays. After practising the conventional PF technique five times, the mid-questionnaire (consisting of the same questions as the pre-questionnaires) and mid-interviews were administered.

\subsubsection{Phase Two}

After administering the mid-questionnaire and mid-interviews, the researcher explained the difference between the conventional form and the new form of the technique. The difference was that participants would provide only macro comments to their fellow students (using the section related to macro features in Alhazmi and Schofield's (2007) evaluation form), and the researcher/teacher would deal with the micro comments. After the difference had been clarified, the participants started developing the first drafts of their sixth essay, which were collected by teacher in the same session and returned in the the following one along with teacher micro comments. After that the participants exchanged essays and started providing only macro PF in pairs. After practising the new form on five essays, identical post-questionnaires and post-interviews were administered. Verbal protocols were recorded when possible during the second phase of the study.

In brief, the participants practised the new form for the same length of time as they practised the conventional use of the PF technique, and their views were investigated both before and after exposure to each type of this technique.

What follows is a review of the results and the analyses, which included a questionnaire, interviews, and verbal protocol recordings.

\section{Results and Analyses}

In order to analyse the quantitative data gathered in this study, descriptive statistics of the questionnaire data were calculated. Additionally, the change in the participants' responses from the pre-questionnaire to the mid-questionnaire (i.e. during their exposure to the conventional form) was measured using a statistical test, and then the change in their responses from the mid-questionnaire to the post-questionnaire (i.e., during exposure to the new form) was measured. This allowed a comparison to be made between the learners' views before and after exposure to each form of the technique. A test of normality was carried out on the questionnaire data, and this showed that the data were not normally distributed; therefore, it was decided that the Wilcoxon Signed 
Ranks Test would be used for the analysis (Larsen-Hall, 2011). Before carrying out this test, negative items in the questionnaire were reverse coded in order to have all the items in a positive direction. Then similar items were combined as one variable.

\subsection{Questionnaire Results}

The Wilcoxon Signed Ranks Test was used to determine whether or not there was a statistically significant difference in the students' preference in the pre-, mid- and post-questionnaires.

\subsubsection{Receiving PF on the Micro Level}

Two items in the questionnaire inquired about the students' preference when it came to receiving PF from their classmates on micro level features. Since the interest of the researcher lay in the theme they reflected rather than in responses to single items, these were combined as a single variable. These items were:

1) When my classmate gives me feedback, I want to see comments indicating errors in spelling and vocabulary.

2) When my classmate gives me feedback, I object to seeing comments indicating errors in grammar and punctuation. (Reverse coded)

Using the SPSS, the test was run (see Table 1 below and Tables A1-A2 in Appendix A) on the pre-questionnaire results $(M=3.96, S D=0.69)$ and the mid-questionnaire results $(M=4.02, S D=0.62)$, and then on the mid-questionnaire and post-questionnaire $(M=3.95, S D=0.67)$ results for all the students in the study, to establish whether or not there were statistically significant changes in their preference when it came to receiving micro level PF from their classmates after each of the two interventions.

Table 1. Students' preferences for micro and macro level PF

\begin{tabular}{llllll}
\hline Stage & $\mathrm{N}$ & Minimum & Maximum & Mean & SD \\
\hline Pre: RCMicroFB & 41 & 2.00 & 5.00 & 3.9634 & .69273 \\
Mid: RCMicroFB & 41 & 2.50 & 5.00 & 4.0244 & .62201 \\
Post: RCMicroFB & 41 & 2.00 & 5.00 & 3.9512 & .67828 \\
Pre: RCMacroFB & 41 & 2.00 & 5.00 & 3.6463 & .67309 \\
Mid: RCMacroFB & 41 & 2.50 & 5.00 & 3.8049 & .54633 \\
Post: RCMacroFB & 41 & 2.50 & 5.00 & 3.9634 & .64605 \\
\hline
\end{tabular}

No statistically significant difference was found $(Z=-0.29, p>0.05)$ between the pre- and mid-questionnaire results. Moreover, no statistically significant difference was found $(Z=0.83, p>0.05)$ between the mid- and post-questionnaire results. Finally, when the pre-and post-questionnaire results were tested, no statistically significant difference was found here either $(Z=0.37, p>0.05)$.

\subsubsection{Receiving PF on the Macro Level}

Two items in the questionnaire inquired about the students' preference when it came to receiving macro level PF from their classmates, and these were also combined as a single variable. These items were:

1) When my classmate gives me feedback, I want to see comments on the organisation of my essay.

2) When my classmate gives me feedback, I object to seeing comments on the ideas I have expressed. (Reverse coded)

A test was run (see Table 1 above and Tables A1-A2 in Appendix A) on the pre-questionnaire results $(M=3.64$, $S D=0.67)$ and the mid-questionnaire results $(M=3.80, S D=0.54)$, and then on the mid-questionnaire and post-questionnaire $(M=3.96, S D=0.64)$ results for all the students in the study, to establish whether or not there were statistically significant changes in their preference with regard to receiving macro level PF from their fellow students after each of the two interventions. No statistically significant difference was found $(Z=-1.09$, $p>0.05)$ between the pre- and mid-questionnaire results. Moreover, no statistically significant difference was found $(Z=-1.52, p>0.05)$ between the mid- and post-questionnaire results. However, when the pre- and post-questionnaire results were compared, a statistically significant difference was found at the level of $p<0.05$ and $Z=-1.99$. 


\subsubsection{Preference for Focusing on the Macro Level}

A test was run (see Table 2 below and Table A3 in Appendix A) on the pre-questionnaire results $(M=2.73, S D=$ $1.04)$ and mid-questionnaire results $(M=2.63, S D=1.01)$, and then on the mid-questionnaire and post-questionnaire $(M=2.70, S D=0.84)$ results for all the students in the study, to establish whether or not there were statistically significant changes in their preference for focusing on the macro level after each of the two interventions.

No statistically significant difference was found $(Z=-0.62, p>0.05)$ between the pre- and mid-questionnaire results. Moreover, no statistically significant difference was found $(Z=-0.20, p>0.05)$ between the mid- and post-questionnaire results.

Table 2. Preferences for one or two levels

\begin{tabular}{llllll}
\hline Stage & $\mathrm{N}$ & Minimum & Maximum & Mean & SD \\
\hline $\begin{array}{l}\text { Pre: I prefer focusing on macro issues when giving } \\
\text { and receiving peer feedback. }\end{array}$ & 41.00 & 5.00 & 2.7317 & 1.04939 \\
$\begin{array}{l}\text { Mid: I prefer focusing on macro issues when } \\
\text { giving and receiving peer feedback. }\end{array}$ & 41 & 1.00 & 5.00 & 2.6341 & 1.01873 \\
$\begin{array}{l}\text { Post: I prefer focusing on macro issues when } \\
\text { giving and receiving peer feedback. }\end{array}$ & 41 & 1.00 & 4.00 & 2.7073 & .84392 \\
$\begin{array}{l}\text { Pre: I prefer focusing on both macro and micro } \\
\text { issues when giving and receiving peer feedback. }\end{array}$ & 41 & 2.00 & 5.00 & 4.2195 & .79095 \\
$\begin{array}{l}\text { Mid: I prefer focusing on both macro and micro } \\
\text { issues when giving and receiving peer feedback. }\end{array}$ & 41 & 2.00 & 5.00 & 4.2195 & .65239 \\
$\begin{array}{l}\text { Post: I prefer focusing on both macro and micro } \\
\text { issues when giving and receiving peer feedback. }\end{array}$ & 41 & 2.00 & 5.00 & 4.1463 & .65425 \\
\hline
\end{tabular}

Additionally, no statistically significant difference was found between the pre- and post-questionnaire results $(Z$ $=-0.11, p=0.91$ ).

\subsubsection{Preference for Focusing on the Macro and Micro Levels}

A test was run (see Table 2 above and Table A3 in Appendix A) on the pre-questionnaire results $(M=4.21, S D=$ $0.79)$ and mid-questionnaire results $(M=4.21, S D=0.65)$, and then on the mid-questionnaire and post-questionnaire $(M=4.14, S D=0.65)$ results for all the students in the study to establish whether there were statistically significant changes in their preference for focusing on either level after each of the two interventions. No statistically significant difference was found $(Z=0.00, p>0.05)$ between the pre- and mid-questionnaire results. Moreover, no statistically significant difference was found $(Z=-0.57, p>0.05)$ between the mid- and post-questionnaire results, and no statistically significant difference was found between the pre- and post-questionnaire results $(Z=-0.39, p=0.69)$.

\subsubsection{Focusing on One Level Encourages Participation in PF}

A test was run (see Table 3 below and Table A4 in Appendix A) on the pre-questionnaire results $(M=2.60, S D=$ $0.91)$ and the mid-questionnaire results $(M=2.97, S D=0.98)$, and then on the mid-questionnaire and post-questionnaire $(M=2.90, S D=1.04)$ results for all the students in the study, to establish whether or not there were statistically significant changes in their beliefs concerning the benefit of focusing on one level after each of the two interventions. 
Table 3. Focusing on one level encourages participation in PF

\begin{tabular}{lllllc}
\hline Stage & $\mathrm{N}$ & Minimum & Maximum & Mean & SD \\
\hline $\begin{array}{l}\text { Pre: Focusing on one level encourages } \\
\text { participation }\end{array}$ & 41 & 1.00 & 4.00 & 2.6098 & .91864 \\
$\begin{array}{l}\text { Mid: Focusing on one level encourages } \\
\text { participation }\end{array}$ & 41 & 1.00 & 5.00 & 2.9756 & .98711 \\
\begin{tabular}{l}
$\begin{array}{l}\text { Post: Focusing on one level encourages } \\
\text { participation in PF }\end{array}$ \\
\hline
\end{tabular} & 41 & 1.00 & 5.00 & 2.9024 & 1.04415 \\
\hline
\end{tabular}

A statistically significant difference was found $(Z=-2.32, p=0.02)$ between the pre- and mid-questionnaire results. However, no statistically significant difference was found $(Z=-0.43, p>0.05)$ between the mid- and post-questionnaire results. Additionally, no statistically significant difference was found between the pre- and post-questionnaire results $(Z=-1.44, p=0.14)$.

\subsubsection{Appreciation of the Way FB Was Given}

A test was run (see Table 4 below and Table A5 in Appendix A) first on the pre-questionnaire results $(M=4.07$, $S D=0.60)$ and the mid-questionnaire results $(M=4.29, S D=0.64)$, and then on the mid-questionnaire and post-questionnaire $(M=4.14, S D=0.61)$ results for all the students in the study, to establish whether or not there were statistically significant changes in their appreciation of the way PF was given by their fellow students after each of the two interventions.

Table 4. Appreciating the way PF was given.

\begin{tabular}{llllll}
\hline Stage & $\mathrm{N}$ & Minimum & Maximum & Mean & $\mathrm{SD}$ \\
\hline $\begin{array}{l}\text { Pre: I appreciated the way that my classmates gave } \\
\text { me written comments on my essays. }\end{array}$ & 41 & 3.00 & 5.00 & 4.0732 & .60788 \\
$\begin{array}{l}\text { Mid: I appreciated the way that my classmates } \\
\text { gave me written comments on my essays. }\end{array}$ & 41 & 2.00 & 5.00 & 4.2927 & .64202 \\
\begin{tabular}{l}
$\begin{array}{l}\text { Post: I appreciated the way that my classmates } \\
\text { gave me written comments on my essays. }\end{array}$ \\
\hline
\end{tabular} & 41 & 2.00 & 5.00 & 4.1463 & .61486 \\
\hline
\end{tabular}

No statistically significant difference was found $(Z=-1.78, p>0.05)$ between the pre- and mid-questionnaire results. Moreover, no statistically significant difference was found $(Z=-1.60, p>0.05)$ between the mid- and post-questionnaire results, and no statistically significant difference was found between the pre- and post-questionnaire results $(Z=-0.61, p=0.53)$.

To conclude, the results indicated that before exposure to each form of PF, and taking into account the standard deviations - i.e., considering the range in the students' overall responses - the students had a greater preference for receiving comments on micro features than for receiving them on macro features. This level of preference for the micro level was maintained, even after exposure to both forms of PF. In addition, their preference for receiving comments on the macro level increased significantly between the pre- and post-questionnaires. These results suggest that the students did not like to focus on the macro level without a similar focus on the micro level. Additionally, the results also show that, before their exposure to both forms of PF, the students did not think that focusing on one level encouraged participation in PF. However, after exposure to both forms, and taking into account the standard deviation, their views seemed to have changed, with their responses giving values both above and below the mid-point on the used scale. Therefore, it can be said that some of the respondents agreed and some disagreed with the notion that focusing on one level encourages participation in PF techniques. Nevertheless, the students appreciated the way in which both the conventional and the new form of $\mathrm{PF}$ were given.

\subsection{Interview Results}

In the mid-interviews, the students reported a preference for focusing on both levels rather than just on the macro level. Various justifications were given for this: so that they could benefit more; to provide more help to others; so that they (macro and micro levels) could complement each other; focusing on one level makes you feel that 
something is missing, and most of the students did not have the ability to critique at the macro level. Additionally, one student reported that, "Focusing on one level led to the deterioration of our essays in the past", as in the past, according to him, teachers mainly paid attention to micro faults.

Only one student reported more interest in macro level PF, and justified this by saying that micro level features can be corrected using word processors and various computer programs. He further claimed to understand the importance of the macro level over the micro level.

On the other hand, the post-interview findings were somewhat surprising. All the students reported preferring to focus on both levels, with the majority showing interest in the second phase of the study where they focused on macro level features. Here, the majority reported several 'disadvantages' resulting from focusing on both levels, and several advantages of focusing on only one level. According to them, some of the problems encountered when focusing on both levels were that students tended to divert most of their attention to the micro level and neglect the macro level; it can be distracting; it doesn't allow the identification of most of the mistakes; it makes for confusion when critiquing; receiving many comments can make you feel disheartened; it can lead to the production of unreliable PF, and lots of pressure is encountered.

Furthermore, focusing on one level (the macro level) allows the students to pay attention to more important aspects of writing; it saves time; it allows them to receive more reliable PF, to produce more in-depth PF, and to avoid weaknesses on the micro level; it does not give the feeling of being distracted; it allows more time for discussion; it encourages more learning; it improves the student's style; it produces an outstanding performance; it reduces the load and gives the feeling of less pressure; it produces constructive PF; it allows the delivery of a clearer message; it permits a deeper analysis of the essay, and it makes the student feel more comfortable.

To conclude, the students preferred to focus on both levels when giving and receiving PF, although they acknowledged the disadvantages of focusing on two levels and the advantages of focusing on one level.

\subsection{Verbal Protocol Recordings}

The findings of the recordings revealed a noticeable phenomenon that seemed to occur in many cases. The students were giving micro PF during the second phase of the study, even though this was deemed to be the teacher's task. The following excerpts illustrate this phenomenon:

Excerpt 1

T (Teacher): Why did you give micro comments?

S1 (Student giving $P F$ ): The micro comments were things that reoccurred, i.e., happened more than once. And it seems that they are owing to force of habit.

Excerpt 2

T: Why did you comment on the grammar?

S1: I think it's because we were used to it. And during the second phase, I couldn't help it. In addition, because there is too much information in the essay, I felt lost and found it difficult to track faults at the macro level; therefore, I tended to shift to locating grammatical and spelling mistakes.

S2 (Student Receiving PF): I agree; we are used to focusing on grammatical mistakes.

Excerpt 3

S1: The spelling in your essay needs to be worked on.

T: Why did you ask about spelling?

S1: Because writers usually commit this mistake. I guess I do it unconsciously, and as a second language learner, you become used to paying attention to spelling. It was pointed out all the time as part of our learning journey. Now it's something in our blood. In other words, I believe it results from the way we were taught over several years.

S2: I noticed that when we read a novel, we don't pay any attention to micro features; the whole of our attention is given to macro features. However, in writing classes, grammar and spelling are commented on.

Next, we present a discussion of the main finings of our quasi-action research study.

\section{Discussion of the Findings}

Knowledge of the learners' standpoints and their perceptions in receiving macro and micro level features in writing were particularly important for this study in order to allow deeper insights into the PF technique to be 
obtained and to enable the researcher to make further recommendations for the future. The questionnaire findings showed that before the learners' exposure to both forms of PF technique, they started off with a preference for receiving PF from their fellow students on micro level as well as on macro level issues. This finding contradicts Hedgcock and Lefkowitz's (1994) argument that ESL students value macro features over micro features and are more concerned with receiving macro FB than micro FB. After practice with both forms of PF, a minor increase was found in the students' preference for receiving PF on both levels, which may indicate that they considered both levels to be almost equally important. This finding supports what they reported in the interviews: both macro and micro level FB complement each other. It may also indicate that they wanted to see comprehensive $\mathrm{PF}$ related to a variety of writing features in order to produce writing of a higher quality at both levels. However, after exposure to the two forms of PF, the students reported a stronger preference for receiving macro comments at a statistically significant level. This can be seen as an indication of their having a deeper understanding of the importance of macro level features, since the participants reported that their previous teachers adopted a traditional teaching perspective (Alhazmi \& Schofield, 2007; Alyousef, 2014; Vassall-Fall, 2011) and prioritised micro features over macro features. In a nutshell, both questionnaire and interviews findings strongly suggested that the participants had a preference for focusing on both levels.

In more detail, in the questionnaires, when the students were asked whether they preferred to focus on macro issues separately when giving and receiving PF, the majority said they did not prefer to receive only macro level PF from their classmates. Although micro FB was supplied by the teacher during practice with the new form of $\mathrm{PF}$, the students did not seem to like being prevented from providing comments to, or receiving them from, their fellow students on this level. It is possible that because the teacher did not offer one-to-one FB sessions and, thus, discussing the FB was not possible, the learners needed someone to collaborate with. A counterpart question was asked in the questionnaire inquiring whether the students would like to give and receive PF based on macro and micro features. The students reported wanting to receive and give PF on the two levels of features both before and after exposure to both forms. When they were asked whether focusing on one level alone encourages participation in PF, in the beginning they stated that it did not, but after they had used the conventional form of PF, a statistically significant improvement was found, reaching the mid-point on the adapted scale. This statistically significant positive change does not mean that they had developed a preference for macro level over micro level PF, but indicates that they had started to acquire a better understanding of the importance of macro features. On the other hand, after exposure to the new form, the participants were less inclined to agree that focusing on one level alone encourages participation in PF. These responses may be indicative of their desire to work with micro features in PF sessions, and perhaps also of their dislike of the idea of the one-level focus.

It is important to speculate on the possible reasons for their desire for the two-level focus from an external point of view, even though they offered several reasons in the interviews. It is possible that they found it difficult to abandon micro features because this is the type of FB they were used to receiving in the past. It is also possible that they may have felt that micro PF was the only type of PF they were likely to succeed in generating and incorporating, and they may also have thought that the focus on macro issues would draw attention to the weaknesses in their writing skills. As a result, they may have thought that if they focused on the macro level, it was likely that the PF would be either of less significance or of no significance at all, a notion that is supported by what they reported in the interviews, which was that most of the students do not have the ability to critique at the macro level. Another possible reason for this is that the participants were very concerned about developing their skills at the micro level and felt that giving and receiving PF on this level would help. Thus, although objective evidence was found of a significant positive change in their learning after exposure to the focus on one level, this was not reflected in their stated preferences.

Although the findings provide clear evidence of the learners' preference for focusing on both levels, the majority of the interviewees in this study reported a greater interest in the second phase of the study (i.e., while practising the new form of PF), where they were asked to focus on macro features when giving and receiving PF. More interestingly, they described several advantages of focusing on the macro level alone, and several disadvantages of focusing on both levels, and yet their preference was for focusing on both levels. This situation may explain their hesitation to accept the new concept of the one-level focus, although it is not clear what caused this hesitation. Did the new form introduce a dramatic change of concept that they found alarming? Should there have been a transitional phase between the uses of the two forms of PF in order to prepare the participants for the newly introduced one-level concept? Unfortunately it is not possible to be certain about this at this stage.

It is interesting that in the interviews the students identified several disadvantages of focusing on both levels, and several advantages of focusing on the macro level alone, and yet clearly stated their preference for focusing on both levels (see the previous Results \& Analysis section). When the students were asked in the questionnaires 
about whether they appreciated the way PF was given, their responses after they had practised both forms of PF were positive. In other words, they did not seem to completely reject or resist the idea of focusing on one level (i.e., the new form of PF) when asked about it indirectly in the questionnaires (i.e., by including the following item: I appreciated the way that my classmates gave me written comments on my essays). As discussed earlier in this section, the students reported not wanting to focus on one level, and this response was given when they were asked directly. Hence, the important question that arises here is: why did students who had acknowledged the advantages of focusing on macro level issues and the disadvantages of focusing on both levels, and who seemed to appreciate the way PF was given in the new form (the one-level form), show resistance to accepting the notion of focusing on one level when asked directly?

This resistance to focusing on macro level issues may be explained by the fact that changing beliefs and perceptions can be difficult. These students had been accustomed to particular teaching methods, and their previous learning experiences had been completely different; thus, radically to change this experience by completely prohibiting the giving and receiving of micro PF (in a PF session) all at once could be a rather aggressive strategy, and could thus lead to rejection. This notion is in line with Benesch (1999) and Turuk (2010), who argue that students might resist the introduction of new thinking in learning contexts. Evidence of this was found during the recording of some of the verbal protocol sessions, when some students started giving micro PF while practising with the new form (when they had been specifically instructed not to give micro PF, which was deemed to be the teacher's task). They were asked what their justifications were for doing something they had been told not to do, and some of the justifications included reasons such as, "I think it's because we were used to it." Another student elaborated further, saying:

Because writers usually commit this mistake. I guess I do it unconsciously, and as a second language learner you become used to paying attention to spelling. It was pointed out all the time during our learning journey. Now it's something in our blood. In other words, I believe it is the result of the way we were taught over several years.

In other words, the traditional methods adopted by their teachers in the past could have been the cause of the students' current difficulties in changing their preferences: i.e., their current beliefs were derived from previous practices.

Moreover, the change found from the questionnaire data in relation to the students' preferences for macro features after each treatment was slight and insignificant. However, when the responses obtained before practice with both forms (i.e., in the pre-questionnaires) were compared to the responses obtained after practice with both forms (i.e., in the post-questionnaires), a statistically significant positive change was found. Although this change does not reflect the participants' preference for focusing on macro features, it does suggest that longer practice can lead to more preference for macro features. This supports our previous argument that the participants were resistant to change, but also suggests that there is a possibility to make a change.

Having discussed the main findings, next we present the conclusion and the suggestions for future research studies.

\section{Conclusion and Suggestions for Future Research}

Investigating learners' preferences concerning any practice may allow deeper insights into it, by which a practitioner can form truthful concepts about his learners and learning in general. This quasi-action research study investigated learners' preferences when it comes to receiving PF from fellow students. Two forms of PF technique were introduced in two consecutive phases: in phase one the students practised providing both macro and micro peer feedback on five essays, while in phase two they provided only macro feedback to their fellow students while the teacher supplied the micro feedback. The participants were 41 EFL undergraduate Saudi students undertaking an English programme at a Saudi higher educational institution. The study adopted a one-group design for the data collection. A mixed method approach was employed using pre-, mid- and post-questionnaires, mid- and post-interviews, and also recording verbal protocol sessions while the participants provided peer feedback to one another. The findings of the study suggested that the participants had a preference for giving and receiving peer feedback on both levels. However, it also appeared that their responses may have been affected by the way they had been taught in the past. Therefore, it is possible to say that learners' preference for receiving macro and micro FB in general is similar regardless of the source of the FB.

The results of the present study have practical implications for future research studies. An important issue that needs to be addressed in future studies is mentioned here. Since we have argued that learners' preferences may change (even though this change can be difficult to make) in the long term, and since the participants in our study acknowledged the advantages of the new form and the disadvantages of the conventional form, and yet retained a preference for the conventional form, an important question that may be asked by researchers, and by 
practitioners in particular is how should we react to learners' preferences? In this regard, as mentioned earlier, the literature does not seem to offer a consensus. For example, Truscott $(1996 ; 1999)$ suggests that learners' preferences should not be taken into account if they are unhelpful to their learning. On the other hand, Bitchener and Ferris (2012) encourage teachers to consider their learners' preferences as this may encourage learner-involvement. We believe that in order to make a decision on this matter, an investigation should be conducted to establish what sort of relationship exists between learner preference and linguistic performance.

Another implication is that since the participants in this study acknowledged the benefit of focusing on one level, while at the same time showing a preference for the two-level form, it may be worthwhile to divide PF sessions into two, so that learners focus on macro level writing features in the first half of the session, while focusing on micro level features in the other half. This would give learners more time to provide PF and also enable them to feel less pressurized. Finally, practising macro level PF may encourage students to have preference for this type of FB, which can indirectly help them develop their proficiency skills in writing.

\section{Acknowledgements}

The authors express their appreciation to the Deanship of Scientific Research at King Saud University and to the Research Center at the Faculty of Arts for funding the current research study.

\section{References}

Alhazmi, S. H., \& Schofield, P. (2007). Enforced revision with checklist and peer feedback in EFL writing: The example of Saudi university students. Scientific Journal of King Faisal University, 8(2), 223-261.

Alyousef, H. (2014). Investigating international postgraduate Business students' multimodal literacy and numeracy practices: A multidimensional appraoch (Unpublished $\mathrm{PhD}$ thesis). University of Adelaide, Adealide.

Benesch, S. (1999). Thinking critically, thinking dialogically. TESOL Quarterly, 33(3), 573-580. http://dx.doi.org/10.2307/3587682

Bitchener, J., \& Ferris, D. (2012). Written corrective feedback in second language acquisition and writing. New York, US: Routledge.

Fazio, L. L. (2001). The effect of corrections and commentaries on the journal writing accuracy of minority- and majority- Language Students. Journal of Second Language Writing, 10(4), 235-249. http://dx.doi.org/10.1016/S1060-3743(01)00042-X

Ferris, D. R. (2004). The "grammar correction" debate in L2 writing: Where are we, and where do we go from here? (and what do we do in the meantime ...?). Journal of Second Language Writing, 13(1), 49-62. http://dx.doi.org/10.1016/j.jslw.2004.04.005

Hedgcock, J., \& Lefkowitz, N. (1994). Feedback on feedback: Assessing learner receptivity to teacher response in L2 composing. Journal of Second Language Writing, 3(2), 141-163. http://dx.doi.org/10.1016/1060-3743(94)90012-4

Kepner, C. G. (1991). An experiment in the relationship of types of written feedback to the development of second-language writing skills. Modern Language Journal, 75(3), 305-313. http://dx.doi.org/10.1111/j.1540-4781.1991.tb05359.x

Larson-Hall, J. (2010). A guide to doing statistics in second language research using SPSS. New York, US: Routledge.

Leki, I. (1991). The preferences of ESL students for error correction in college-level writing classes. Foreign Language Annals, 24(3), 203-218. http://dx.doi.org/10.1111/j.1944-9720.1991.tb00464.x

Min, H. T. (2006). The effects of trained peer review on EFL students' revision types and writing quality. Journal of Second Language Writing, 15(2), 118-141. http://dx.doi.org/10.1016/j.jslw.2006.01.003

Sheppard, K. (1992). Two feedback types: Do they make a difference? RELC Journal, 23(1), 103-110. http://dx.doi.org/10.1177/003368829202300107

Truscott, J. (1996). The case against grammar correction in L2 writing classes. Language Learning, 46(2), 327-369. http://dx.doi.org/10.1111/j.1467-1770.1996.tb01238.x

Truscott, J. (1999). The case for "the case against grammar correction in L2 writing classes": A response to Ferris. Journal of Second Language Writing, 8(2), 111-122. http://dx.doi.org/10.1016/S1060-3743 (99)80124-6 
Turuk, K. M. (2010). Developing critical thinking skills through integrative teaching of reading and writing in the L2 writing classroom (Unpublished $\mathrm{PhD}$ thesis). Newcastle University, Newcastle. Retrieved from https://theses.ncl.ac.uk/dspace/handle/10443/1063

Vassall-Fall, D. (2011). Arab students' perceptions of strategies to reduce memorization. AWEJ, 2, 48-69. Retrieved from http://hdl.handle.net/123456789/18247

Zhao, H. (2010). Investigating learners' use and understanding of peer and teacher feedback on writing: A comparative study in a Chinese English writing classroom. Assessing Writing, 15(1), 3-17. http://dx.doi.org/10.1016/j.asw.2010.01.002

\section{Appendix A}

Table A1. Test Statistics ${ }^{\mathrm{c}}$ (preferences for micro and macro levels)

\begin{tabular}{lllll}
\hline & $\begin{array}{l}\text { MidRCMicroFB- } \\
\text { PreRCMicroFB }\end{array}$ & $\begin{array}{l}\text { PostRCMicroFB- } \\
\text { MidRCMicroFB }\end{array}$ & $\begin{array}{l}\text { MidRCMacroFB- } \\
\text { PreRCMacroFB }\end{array}$ & $\begin{array}{l}\text { PostRCMacroFB- } \\
\text { MidRCMacroFB }\end{array}$ \\
\hline $\mathrm{Z}$ & $-.293-^{\mathrm{a}}$ & $-.83-^{\mathrm{b}}$ & $-1.099-^{\mathrm{a}}$ & $-1.528-^{\mathrm{a}}$ \\
Asymp. Sig. (2-tailed) & .770 & .405 & .272 & .127 \\
\hline
\end{tabular}
a. Based on negative ranks.
b. Based on positive ranks.
c. Wilcoxon Signed Ranks Test.

Table A2. Test Statistics ${ }^{\mathrm{c}}$ (preferences for micro and macro levels)

\begin{tabular}{lll}
\hline & $\begin{array}{l}\text { PostRCMicroFB- } \\
\text { PreRCMicroFB }\end{array}$ & $\begin{array}{l}\text { PostRCMacroFB- } \\
\text { PreRCMacroFB }\end{array}$ \\
\hline Z & $-.372^{\mathrm{a}}$ & $-1.997-^{\mathrm{b}}$ \\
Asymp. Sig. (2-tailed) & .710 & .046 \\
\hline
\end{tabular}
a. Based on positive ranks.
b. Based on negative ranks.
c. Wilcoxon Signed Ranks Test.

Table A3. Test Statistics ${ }^{\mathrm{d}}$ (preferences for one or two levels)

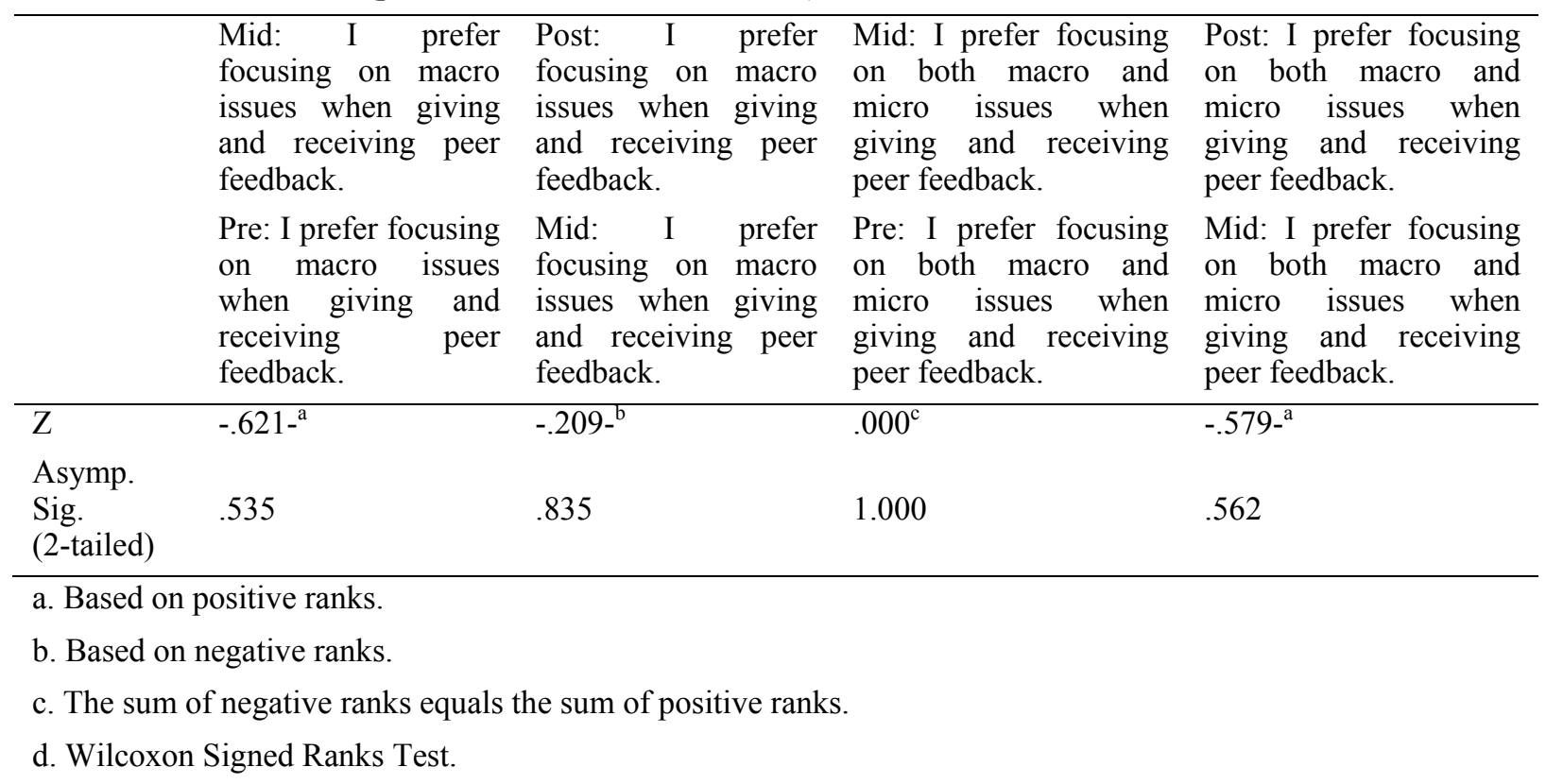


Table A4. Test Statistics ${ }^{\mathrm{c}}$ (focusing on one level encourages participation in PF)

\begin{tabular}{|c|c|c|}
\hline & $\begin{array}{l}\text { Mid: Focusing on one level } \\
\text { encourages participation. }\end{array}$ & $\begin{array}{l}\text { Post: Focusing on one level } \\
\text { encourages participation in PF. }\end{array}$ \\
\hline & $\begin{array}{l}\text { Pre: Focusing on one level } \\
\text { encourages participation. }\end{array}$ & $\begin{array}{l}\text { Mid: Focusing on one level } \\
\text { encourages participation. }\end{array}$ \\
\hline $\mathrm{Z}$ & $-2.327-^{\mathrm{a}}$ & $-.430-^{b}$ \\
\hline Asymp. Sig. (2-tailed) & .020 & .667 \\
\hline
\end{tabular}
a. Based on negative ranks.
b. Based on positive ranks.
c. Wilcoxon Signed Ranks Test.

Table A5. Test Statistics ${ }^{\mathrm{c}}$ (appreciating the way PF was given)

\begin{tabular}{lll}
\hline & $\begin{array}{l}\text { Mid: I appreciated the way that } \\
\text { my classmates gave me written } \\
\text { comments on my essays. }\end{array}$ & $\begin{array}{l}\text { Post: I appreciated the way that } \\
\text { my classmates gave me written } \\
\text { comments on my essays. }\end{array}$ \\
& $\begin{array}{l}\text { Pre: I appreciated the way that } \\
\text { my classmates gave me written } \\
\text { comments on my essays. }\end{array}$ & $\begin{array}{l}\text { Mid: I appreciated the way that } \\
\text { my classmates gave me written } \\
\text { comments on my essays. }\end{array}$ \\
\hline Z & $-1.784-^{\mathrm{a}}$ & $-1.604-^{\mathrm{b}}$ \\
Asymp. Sig. (2-tailed) & .074 & .109 \\
\hline
\end{tabular}

a. Based on negative ranks.

b. Based on positive ranks.

c. Wilcoxon Signed Ranks Test.

\section{Copyrights}

Copyright for this article is retained by the author(s), with first publication rights granted to the journal.

This is an open-access article distributed under the terms and conditions of the Creative Commons Attribution license (http://creativecommons.org/licenses/by/3.0/). 